\title{
Estrategias para la mejora de la cultura organizacional de Servicios Analíticos Generales S.A.C. aplicando el modelo de Denison
}

RECIBIDO: 07/12/2017 ACEPTADO: 14/05/2018

Carmen Balcazar Guerra ${ }^{1}$

\begin{abstract}
RESUMEN
El problema presentado en esta investigación se dividió en las 4 dimensiones según el modelo de Denison para la medición de la cultura organizacional, de la empresa de servicios de laboratorio Servicios Analíticos Generales S.A.C. Cuando se aplica el cuestionario según las dimensiones se aprecia que es necesario el uso de estrategias para la mejora de la cultura organizacional.

Al aplicar las estrategias de liderazgo y motivación, se aprecia el cambio en la cultura organizacional, permitiendo que el personal de operaciones se integre al administrativo.
\end{abstract}

Palabras-claves: Cultura organizacional; involucramiento; consistencia; adaptabilidad y misión.

STRATEGIES FOR THE IMPROVEMENT OF THE ORGANIZATIONAL CULTURE OF GENERAL ANALYTICAL SERVICES S.A.C. APPLYING THE DENISON MODEL

\section{ABSTRACT}

The problem presented in this research was divided into the 4 dimensions according to Denison's model for the measurement of the organizational culture of the laboratory services company Servicios Analíticos Generales S.A.C. When the questionnaire is applied according to the dimensions, it is seen that it is necessary to use strategies to improve the organizational culture.

For applying the leadership and motivation strategies, the change in the organizational culture is appreciated, allowing the operations personnel to be integrated into the administrative.

Keywords: Organizational Culture; Involvement; Consistency; Adaptability and Mission.

\section{INTRODUCCIÓN}

La cultura organizacional concibe como un sistema particular de símbolos, influido por la sociedad circundante, por la historia de la organización y por sus líderes pasados, así como también por diferentes factores de contingencia tales como la tecnología, el mercado y la competencia (Calderón, Murillo y Torres, 2003).

A los elementos ya mencionados de valores, creencias y principios, Denison agrega otro factor a la cultura organizacional: "el conjunto de procedimientos, y conductas gerenciales que sirven de ejemplo y refuerzan esos principios básicos" (Denison, 1991).

El modelo de Denison (Bonavia, Prado y García-Hernández, 2010) es un instrumento construido tras una revisión profunda de la investigación acerca de cómo la cultura influye en la efectividad organizacional (Denison, 2001). Estos valores se agrupan en cuatro rasgos culturales o dimensiones para medir la cultura organizacional de una empresa; en el caso de Servicios Analíticos Generales S.A.C., se disgregaron los problemas en las 4 dimensiones de involucramiento, consistencia, adaptabilidad y misión. Siendo lo más resaltante al aplicar el cuestionario en el personal la falta de liderazgo y motivación.

En este estudio se van aplicar estrategias para la mejora de la cultura organizacional de un laboratorio de servicios. La empresa Servicios Analíticos Generales S.A.C., es un Laboratorio de Análisis y Monitoreo Ambiental reconocido por INACAL-DA bajo la norma NTP - ISO / IEC 17025:2006, con registro No LE-047. Comprometido con el Medio Ambiente, garantizando resultados analíticos confiables y oportunos, enfocados en el cumplimiento de las normativas legales vigentes, y aplicando metodologías estandarizadas según normas EPA, NTP, APHA, ASTM e ISO (Servicios Analíticos Generales, 2016).

1 Licenciada en Química, E-mail: kth_lina@hotmail.com 
El objetivo de la investigación es determinar en qué medida la implementación de las estrategias de liderazgo y motivación mejora los niveles de la cultura organizacional según el modelo de Denison en Servicios Analíticos Generales S.A.C.

La justificación metodológica del estudio se presenta, porque el proyecto por realizar propone nuevas estrategias para generar conocimiento válido y confiable, lo cual ayudará a mejorar la cultura organizacional.

Se concluye que las estrategias utilizadas si tuvieron efecto en la mejora de la cultura organizacional de la organización.

\section{METODOLOGÍA}

El diseño utilizado en la investigación es pre-experimental porque no tienen la capacidad de controlar adecuadamente los factores que influyen contra la validez interna así como también de la validez externa. Sin embargo, este tipo de diseño se utiliza por 2 razones: 1.- Porque son elementos de los diseños experimentales y 2.- Porque ilustran la forma en que las variables extrañas pueden influir en la validez interna (principalmente) de un diseño (Sánchez y Reyes, 2006)

El total de trabajadores son 108 personas pertenecientes a 2 grupos; administrativos y operaciones; dentro de operaciones se divide en laboratorio y monitoreo.

Asimismo, para la medición de la cultura organizacional se ha utilizado el cuestionario validado por Bonavia et al. (2010) adaptado de Denison.

Se realizaron 4 talleres que se detallados a continuación, motivación, liderazgo, trabajo en equipo y cultura organizacional.

Para verificar si existe relación entre la cultura organizacional y las estrategias de mejora se plantearon 4 hipótesis, las cuales se verificaron con las herramientas estadísticas conocidas y muy usadas para estos tipos de datos.

Se aplicaron encuestas antes y después de aplicar las estrategias realizándose las medidas correspondientes.

Así mismo en el Tabla 1, se tiene la distribución de las preguntas de la encuesta.
Tabla 1. Distribución de preguntas en la encuesta Denison.

\begin{tabular}{|l|l|c|}
\hline Características & \multicolumn{1}{|c|}{ Índices } & $\begin{array}{c}\mathbf{N}^{\circ} \text { de } \\
\text { preguntas }\end{array}$ \\
\hline \multirow{3}{*}{ Involucramiento } & Empoderamiento & 1 al 5 \\
\cline { 2 - 3 } & Trabajo en equipo & 6 al 10 \\
\cline { 2 - 3 } & Desarrollo de capacidades & 11 al 15 \\
\hline \multirow{4}{*}{ Adaptabilidad } & Valores Centrales & 16 al 20 \\
\cline { 2 - 3 } & Acuerdo & 21 al 25 \\
\cline { 2 - 3 } & Coordinación e Integración & 26 al 30 \\
\hline \multirow{4}{*}{ Misión } & Creación del cambio & 31 al 35 \\
\cline { 2 - 3 } & Enfoque en el cliente & 36 al 40 \\
\cline { 2 - 3 } & Aprendizaje organizacional & 41 al 45 \\
\hline & Dirección y propósitos estra- & 46 al 50 \\
\cline { 2 - 3 } & tégicos & 51 al 55 \\
\cline { 2 - 3 } & Metas y objetivos & 56 al 60 \\
\cline { 2 - 3 } & Visión & \\
\hline
\end{tabular}

Fuente. Datos tomados de Barreto y Bonilla (2011).

Las preguntas fueron medidas bajo el diseño de la escala de Likert, el cual se le considera escala de intervalo porque tiene de 5 a 7 categorías, y al comparar las respuestas de los encuestados se notará la diferencia mental entre ellas, por lo que la escala viene siendo más que ordinal ya que se pueden encontrar distancias (De la Garza et al., 2013). Se encontrará en la Tabla 2 líneas abajo.

Tabla 2. Escala de Likert.

\begin{tabular}{|c|c|c|c|c|}
\hline $\begin{array}{c}\text { Muy en } \\
\text { desacuerdo }\end{array}$ & $\begin{array}{c}\text { En } \\
\text { desacuerdo }\end{array}$ & Neutral & $\begin{array}{c}\text { De } \\
\text { acuerdo }\end{array}$ & $\begin{array}{c}\text { Totalmente } \\
\text { de acuerdo }\end{array}$ \\
\hline 1 & 2 & 3 & 4 & 5 \\
\hline
\end{tabular}

Fuente. Datos tomados de Barreto y Bonilla (2011).

\section{RESULTADOS Y DISCUSIÓN}

Para determinar el número de trabajadores de Servicios Analíticos Generales S.A.C a ser considerados para la muestra; Quintero, Africano y Faría (2008) sostienen que se utilizó la fórmula de Sierra (2003), la cual se aplica para el caso de poblaciones finitas menores de 100.000 (Hospinal 2014, p. 63), representada por el siguiente estadístico:

$$
n=\frac{4 \times N \times p \times q}{E^{2}(N-1)+4 \times p \times q}
$$

Donde el significado de cada una de las expresiones, son las siguientes:

$$
\begin{aligned}
& n=\text { tamaño de la muestra } \\
& E^{2}=\text { error seleccionado por el investigador } \\
& 4=\text { constante } \\
& p=\text { probabilidad de éxito representado por el } \\
& 50 \%=0,50 \\
& q=\text { probabilidad de fracaso con un valor de } 50 \% \\
& =0,50 \\
& N=\text { tamaño de la población }
\end{aligned}
$$


Según la fórmula redondeando se tiene que encuestar a 86 trabajadores de la empresa.

Como el total es de 86 colaboradores, se divide en 2 grupos como se indica a continuación:

Se estratifica de $N=108, n=86,43$ y 43

Administrativos $=33.33 \% \quad 14,33$ redondeando 14 colab.

Operativos $=66.66 \% \quad 8,66$ redondeando 29 colab .

-43 Colaboradores GE:

-43 Colaboradores GC:

GC: Grupo control y GE: Grupo experimental

\section{HIPÓTESIS ANTES Y DESPUÉS DE LA IMPLE- MENTACIÓN DE LAS ESTRATEGIAS}

1. Hipótesis específica 1: Mediante la implementación de talleres de inducción de la cultura organizacional y dinámica para reflexionar sobre el liderazgo se logrará la mejora de la dimensión involucramiento de la cultura organizacional según el modelo de Denison en Servicios Analíticos Generales S.A.C.

En los Tablas 3 y 7 se puede encontrar los valores de las encuestas realizadas al área de administración y operaciones respectivamente de la dimensión involucramiento, como se aprecia las estrategias ha tenido un efecto positivo.

Tabla 3. Área administrativa antes y después de la estrategia (involucramiento, pre test y post test)

\begin{tabular}{|c|c|c|}
\hline Colaboradores & $\begin{array}{c}\text { Involucramiento } \\
\text { pre-ad }\end{array}$ & $\begin{array}{c}\text { Involucramiento } \\
\text { post-ad }\end{array}$ \\
\hline 1 & 53 & 64 \\
\hline 2 & 49 & 59 \\
\hline 3 & 53 & 69 \\
\hline 4 & 48 & 57 \\
\hline 5 & 48 & 61 \\
\hline 6 & 40 & 55 \\
\hline 7 & 42 & 57 \\
\hline 8 & 39 & 54 \\
\hline 9 & 46 & 59 \\
\hline 10 & 29 & 49 \\
\hline 11 & 45 & 59 \\
\hline 12 & 47 & 62 \\
\hline 13 & 44 & 59 \\
\hline 14 & 47 & 57 \\
\hline
\end{tabular}

Fuente. Elaboración Propia.
Se utiliza el Minitab como herramienta para tratar los datos del pre test y post test para los estadísticos descriptivos del el área administrativa (Tabla 4 y Tabla 5) y de operaciones (Tabla 8 y Tabla 9).

Tabla 4. Determinación de los datos con estadística descriptiva (involucramiento, pre test administrativos)

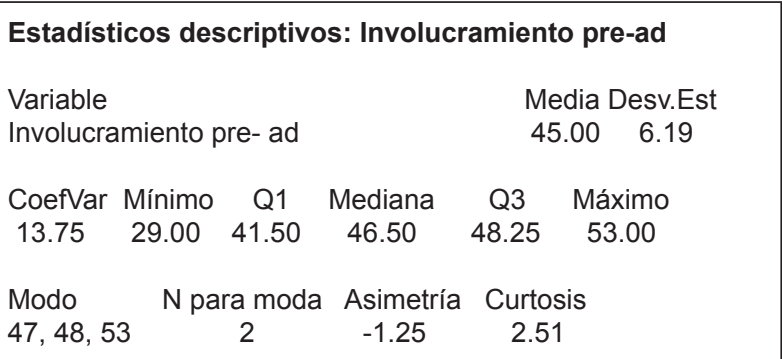

Fuente. Elaboración Propia.

Tabla 5. Determinación de los datos con estadística descriptiva (involucramiento, post test administrativos)

\begin{tabular}{|c|c|c|c|c|c|}
\hline \multicolumn{4}{|l|}{ Variable } & Media & Desv.Est \\
\hline \multicolumn{4}{|c|}{ Involucramiento post - ad } & 58.64 & 4.72 \\
\hline CoefVar & Mínimo & Q1 & Mediana & Q3 & \\
\hline 8.04 & 49.00 & 56.50 & 59.00 & 1.25 & \\
\hline \multicolumn{3}{|c|}{ Máximo Modo N para moda } & Asimetría & Curtosis & \\
\hline 69.00 & 9 & 4 & 0.22 & 1.55 & \\
\hline
\end{tabular}

Fuente. Elaboración Propia.

Como ambas distribuciones son normales se aplica la t- pareada (t-student) para comparar las mediciones antes y después de aplicar las estrategias. Esto se puede apreciar en los Tablas 6 que se detalla líneas abajo.

Tabla 6. Prueba de t-student

Límite superior 95\% para la diferencia de la
media: -12.259
Prueba $t$ de diferencia media $=0$ (vs. < 0$):$
Valor $\mathrm{T}=-17.45$ valor $\mathrm{p}=0.0$

Fuente. Elaboración Propia.

En el Tabla 6 al comparar las 2 mediciones antes y después, según la hipótesis si p es mayor o igual a 0.05 se acepta $\mathrm{H}_{0}$ si las medianas son iguales y se rechaza si $\mathrm{H}_{1}: \mu_{1}<\mu_{2}$; en este caso el valor de $p=0.0$, por lo tanto se rechaza la hipótesis nula y se acepta la alternativa. 
Tabla 7. Área operacional antes y después de la estrategia (involucramiento, pre test y post test)

\begin{tabular}{|c|c|c|}
\hline Colaboradores & $\begin{array}{c}\text { Involucramiento } \\
\text { pre-ope }\end{array}$ & $\begin{array}{l}\text { Involucramiento } \\
\text { post-ope }\end{array}$ \\
\hline 1 & 39 & 54 \\
\hline 2 & 47 & 60 \\
\hline 3 & 65 & 69 \\
\hline 4 & 49 & 65 \\
\hline 5 & 49 & 64 \\
\hline 6 & 37 & 52 \\
\hline 7 & 32 & 47 \\
\hline 8 & 66 & 68 \\
\hline 9 & 61 & 65 \\
\hline 10 & 47 & 61 \\
\hline 11 & 42 & 56 \\
\hline 12 & 42 & 57 \\
\hline 13 & 65 & 66 \\
\hline 14 & 45 & 59 \\
\hline 15 & 42 & 57 \\
\hline 16 & 54 & 59 \\
\hline 17 & 63 & 68 \\
\hline 18 & 47 & 61 \\
\hline 19 & 44 & 60 \\
\hline 20 & 39 & 53 \\
\hline 21 & 40 & 55 \\
\hline 22 & 49 & 61 \\
\hline 23 & 65 & 67 \\
\hline 24 & 51 & 60 \\
\hline 25 & 59 & 65 \\
\hline 26 & 53 & 53 \\
\hline 27 & 58 & 62 \\
\hline 28 & 30 & 49 \\
\hline 29 & 42 & 53 \\
\hline
\end{tabular}

Fuente. Elaboración Propia.

Con los datos de la tabla 7 se tiene los datos estadísticos detallados en la tabla 8 y 9 líneas abajo.

Tabla 8. Determinación de los datos con estadística descriptiva (involucramiento, pre test operaciones)

\begin{tabular}{|c|c|c|c|c|}
\hline \multicolumn{5}{|c|}{ Estadísticos descriptivos: Involucramiento pre-ope } \\
\hline \multirow{2}{*}{\multicolumn{3}{|c|}{$\begin{array}{l}\text { Variable } \\
\text { Involucramiento pre - op }\end{array}$}} & \multicolumn{2}{|l|}{ Media } \\
\hline & & & 49.03 & \\
\hline $\begin{array}{l}\text { Desv.Est } \\
10.26\end{array}$ & $\begin{array}{c}\text { CoefVar } \\
20.02\end{array}$ & $\begin{array}{l}\text { Mínimo } \\
30.00\end{array}$ & $\begin{array}{c}\text { Q1 } \\
42.00\end{array}$ & $\begin{array}{c}\text { Mediana } \\
47.00\end{array}$ \\
\hline $\begin{array}{c}\text { Q3 } \\
58.50\end{array}$ & $\begin{array}{l}\text { Máximo } \\
66.00\end{array}$ & $\begin{array}{l}\text { Modo } \\
42\end{array}$ & $\begin{array}{c}\text { N para moda } \\
4\end{array}$ & $\begin{array}{c}\text { Asimetría } \\
0.19\end{array}$ \\
\hline $\begin{array}{l}\text { Curtosis } \\
-0.84\end{array}$ & \multicolumn{3}{|c|}{ Curtosis } & \\
\hline
\end{tabular}

Fuente. Elaboración Propia.
Tabla 9. Determinación de los datos con estadística descriptiva (involucramiento, post test operaciones)

\begin{tabular}{|lccccc}
\hline \multicolumn{4}{l}{ Estadísticos descriptivos: Involucramiento post-ope } \\
Variable & & Media & Desv.Est \\
Involucramiento post - op & 59.52 & 5.93 & \\
& & & & \\
CoefVar & Mínimo & Q1 & Mediana & Q3 & Máximo \\
9.96 & 47.00 & 54.50 & 60.00 & 65.00 & 69.00 \\
& & & & & \\
Modo & & N para moda & Asimetría & \\
$53,60,61,65$ & 3 & & -0.25 & \\
& & & & & \\
Curtosis & & & & \\
-0.71 & & &
\end{tabular}

Fuente. Elaboración Propia.

Como ambas distribuciones son normales se aplica la t-student (Montgomery, 2015) luego la t-pareada para comparar las mediciones antes y después de aplicar las estrategias (Bower, 2000). Esto se puede apreciar en la Tabla 10 que se detalla líneas abajo.

Tabla 10. Prueba de t-student

Límite superior $95 \%$ para la diferencia de la media: -8.30

Prueba t de diferencia media $=0$ (vs. $<0$ ): Valor $\mathrm{T}=-9.37$ Valor $p=0.0$

Fuente. Elaboración Propia.

Como se puede apreciar el p value es igual a cero, entonces es menor que 0.05 , por lo cual se rechaza la hipótesis nula y se acepta a hipótesis alternativa.

$\mathrm{H}_{\mathrm{o}}: \mu 1=\mu 2, \mathrm{H}_{1}: \mu 1<\mu 2$, entonces la media del involucramiento pre test es menor que la media del involucramiento post test.

Por lo tanto, hay evidencia suficiente para aceptar la hipótesis específica 1 , se cumple tanto para los colaboradores administrativos y de operaciones.

2. Hipótesis específica 2: Mediante la implementación de dinámica de motivación en el trabajo se logrará la mejora de la dimensión consistencia de la cultura organizacional según el modelo de Denison en Servicios Analíticos Generales S.A.C.

En las Tablas 11 y 15 se puede encontrar los valores de las encuestas realizadas al área de administración y de operaciones respectivamente de la dimensión consistencia, como se aprecia las estrategias ha tenido un efecto positivo. Se aplica en el Minitab los datos del pre test y post 
test para los estadísticos descriptivos para el área administrativa (Tabla 12 y Tabla 13) y de operaciones (Tabla 16 y Tabla 17).

Tabla 11. Área administrativa antes y después de la estrategia (consistencia, pre test y post test)

\begin{tabular}{|c|c|c|}
\hline $\begin{array}{c}\text { Colaboradores pre } \\
\text { test }\end{array}$ & $\begin{array}{c}\text { Consistencia } \\
\text { pre-ad }\end{array}$ & $\begin{array}{c}\text { Consistencia } \\
\text { post-ad }\end{array}$ \\
\hline 1 & 43 & 58 \\
\hline 2 & 43 & 57 \\
\hline 3 & 47 & 67 \\
\hline 4 & 43 & 58 \\
\hline 5 & 45 & 60 \\
\hline 6 & 43 & 58 \\
\hline 7 & 39 & 54 \\
\hline 8 & 44 & 58 \\
\hline 9 & 39 & 58 \\
\hline 10 & 27 & 51 \\
\hline 11 & 39 & 58 \\
\hline 12 & 40 & 55 \\
\hline 13 & 43 & 58 \\
\hline 14 & 50 & 60 \\
\hline
\end{tabular}

Fuente. Elaboración Propia.

Tabla 12. Determinación de los datos con estadística descriptiva (consistencia, pre test administrativos)

\begin{tabular}{|lccccc|}
\hline \multicolumn{4}{ll}{ Estadísticos descriptivos: Consistencia pre-ad } \\
Variable & & Media & Desv.Est \\
Consistencia pre- ad & 41.79 & 5.28 & \\
& & & & & \\
CoefVar & Mínimo & Q1 & Mediana & Q3 & Máximo \\
12.63 & 27.00 & 39.00 & 43.00 & 4.25 & 50.00 \\
& & & & & \\
Modo & N para moda & Asimetría & Curtosis \\
43 & 5 & -1.56 & 4.57 \\
\hline
\end{tabular}

Fuente. Elaboración Propia.

Tabla 13. Determinación de los datos con estadística descriptiva (consistencia, post- test administrativos)

\begin{tabular}{|lccccc|}
\hline \multicolumn{4}{l}{$\begin{array}{l}\text { Estadísticos descriptivos: } \\
\text { Variable }\end{array}$} & & Consistencia post-ad \\
Consistencia post - ad & 57.857 & 3.549 & Desv.Est \\
& & & & & \\
CoefVar & Mínimo & Q1 & Mediana & Q3 & Máximo \\
6.13 & 51.00 & 56.50 & 58.00 & 58.50 & 67.00 \\
& & & & & \\
Modo & N para moda & Asimetría & Curtosis \\
58 & 7 & 0.76 & 3.51 \\
\hline
\end{tabular}

Fuente. Elaboración Propia.

Con la dimensión consistencia, al realizar la prueba de normalidad, se obtiene distribución no nor- mal, por esta razón se utiliza la prueba de Wilcoxon Mann Whitney (Siegel y Castellan, 2009), para comparar las medianas, se detalla en el Tabla 14 líneas abajo.

Tabla 14. Prueba de Wilcoxon Mann Whitney

Prueba de Mann-Whitney e IC: Consistencia pre-ad, Consistencia post-ad

$\begin{array}{lcc} & \text { N } & \text { Mediana } \\ \text { Consistencia pre-ad } & 14 & 43.000 \\ \text { Consistencia post-ad } & 14 & 58.000\end{array}$

La estimación del punto para $\eta 1$ - $\eta 2$ es -15.000

95.4 El porcentaje IC para $\eta 1$ - $\eta 2$ es $(-19.000,-12.999)$

$\mathrm{W}=105.0$

Prueba de $\eta 1=\eta 2$ vs. $\eta 1<\eta 2$ es significativa en 0.0000 La prueba es significativa en 0.0000 (ajustado por empates)

Fuente. Elaboración Propia.

Como se puede apreciar el nivel de significancia es igual a cero entonces se rechaza la hipótesis nula y se acepta a hipótesis alternativa.

Tabla 15. Área operacional antes y después de la estrategia (consistencia, pre test y post test)

\begin{tabular}{|c|c|c|}
\hline Colaboradores & $\begin{array}{c}\text { Consistencia } \\
\text { pre-op }\end{array}$ & $\begin{array}{c}\text { Consistencia } \\
\text { post-op }\end{array}$ \\
\hline 1 & 36 & 49 \\
\hline 2 & 51 & 51 \\
\hline 3 & 58 & 63 \\
\hline 4 & 45 & 59 \\
\hline 5 & 43 & 56 \\
\hline 6 & 40 & 55 \\
\hline 7 & 35 & 49 \\
\hline 8 & 57 & 62 \\
\hline 9 & 50 & 63 \\
\hline 10 & 42 & 56 \\
\hline 11 & 48 & 63 \\
\hline 12 & 41 & 56 \\
\hline 13 & 58 & 61 \\
\hline 14 & 37 & 52 \\
\hline 15 & 40 & 55 \\
\hline 16 & 40 & 58 \\
\hline 17 & 43 & 58 \\
\hline 18 & 42 & 57 \\
\hline 19 & 44 & 55 \\
\hline 20 & 35 & 50 \\
\hline 21 & 41 & 52 \\
\hline 22 & 48 & 63 \\
\hline 23 & 56 & 62 \\
\hline 24 & 45 & 60 \\
\hline 25 & 45 & 60 \\
\hline 26 & 43 & 43 \\
\hline 27 & 55 & 62 \\
\hline 28 & 37 & 55 \\
\hline 29 & 36 & 51 \\
\hline
\end{tabular}

Fuente. Elaboración Propia. 
$H_{0}: \mu 1=\mu 2, H_{1}: \mu 1<\mu 2$, entonces la mediana de consistencia pre test es menor que la media de consistencia post test. Por lo tanto, hay evidencia suficiente para aceptar la hipótesis específica 2.

Tabla 16. Determinación de los datos con estadística descriptiva (consistencia, pre test operaciones)

\begin{tabular}{|lccccc|}
\hline \multicolumn{4}{ll}{ Estadísticos descriptivos: Consistencia pre-op } \\
Variable & & Media & Desv.Est & \\
Consistencia pre- op & 44.38 & 7.27 & \\
& & & & & \\
CoefVar & Mínimo & Q1 & Mediana & Q3 & Máximo \\
16.38 & 33.00 & 40.00 & 43.00 & 49.00 & 58.00 \\
& & & & & \\
Modo & N para moda & Asimetría & Curtosis \\
$40,43,45$ & 3 & 0.53 & -0.57 \\
\hline
\end{tabular}

Fuente. Elaboración Propia.

Tabla 17. Determinación de los datos con estadística descriptiva (consistencia, post test operaciones)

\begin{tabular}{|lccccc|}
\hline \multicolumn{4}{ll}{ Estadísticos descriptivos: Consistencia post-ad } \\
Variable & & Media & Desv.Est & \\
Consistencia & post- op & 56.414 & 5.220 & \\
& & & & & \\
CoefVar & Mínimo & Q1 & Mediana & Q3 & Máximo \\
9.25 & 43.00 & 52.00 & 56.00 & 61.50 & 63.00 \\
& & & & & \\
Modo & N para moda & Asimetría & Curtosis \\
55,63 & 4 & -0.57 & -0.14 \\
\hline
\end{tabular}

Fuente. Elaboración Propia.

Como ambas distribuciones son normales se aplica la t- pareada (t-student) para comparar las mediciones antes y después de aplicar las estrategias. Esto se puede apreciar en la tabla 18 que se detalla líneas abajo.

Tabla 18. Prueba de $t$-student

Límite superior 95\% para la diferencia de la media: -9.883
$\begin{aligned} & \text { Prueba t de diferencia media }=0(\text { vs. }<0) \text { : Valor } \mathrm{T}=-11.79 \\ & \text { Valor } p=0.0\end{aligned}$

Fuente. Elaboración Propia.

Como se puede apreciar el p value es igual a cero, entonces por lo tanto es menor que 0.05 (Ciencia y educación, 2016), entonces se rechaza la hipótesis nula y se acepta a hipótesis alternativa .
$H_{0}: \mu 1=\mu 2, H_{1}: \mu 1<\mu 2$, entonces la media de consistencia pre test es menor que la media del involucramiento post test. Por lo tanto, hay evidencia suficiente para aceptar la hipótesis específica 2 tanto para los colaboradores administrativos y de operaciones.

3. Hipótesis específica 3: Mediante la implementación de los talleres de motivación en el trabajo se logrará la mejora de la dimensión adaptabilidad de la cultura organizacional según el modelo de Denison en Servicios Analíticos Generales S.A.C.

En las Tablas 19 y 23 se puede encontrar los valores de las encuestas realizadas al área de administración y de operaciones respectivamente de la dimensión adaptabilidad. Se aplica en el Minitab los datos del pre test y post test para los estadísticos descriptivos para el área administrativa (Tabla 20 y Tabla 21) y de operaciones (Tabla 24 y Tabla 25).

Tabla 19. Área administrativa antes y después de la estrategia (adaptabilidad, pre test y post test)

\begin{tabular}{|c|c|c|}
\hline Colaboradores & $\begin{array}{c}\text { Adaptabilidad } \\
\text { pre-ad }\end{array}$ & $\begin{array}{c}\text { Adaptabilidad } \\
\text { post-ad }\end{array}$ \\
\hline 1 & 43 & 58 \\
\hline 2 & 46 & 59 \\
\hline 3 & 56 & 69 \\
\hline 4 & 42 & 57 \\
\hline 5 & 50 & 64 \\
\hline 6 & 41 & 56 \\
\hline 7 & 40 & 55 \\
\hline 8 & 39 & 57 \\
\hline 9 & 40 & 55 \\
\hline 10 & 39 & 57 \\
\hline 11 & 40 & 55 \\
\hline 12 & 41 & 56 \\
\hline 13 & 40 & 55 \\
\hline 14 & 54 & 63 \\
\hline
\end{tabular}

Fuente. Elaboración Propia.

Tabla 20. Determinación de los datos con estadística descriptiva (adaptabilidad, pre test administrativos)

\begin{tabular}{|c|c|c|c|c|c|}
\hline \multicolumn{6}{|c|}{ Estadísticos descriptivos: adaptabilidad pre-ad } \\
\hline \multicolumn{2}{|l|}{ Variable } & \multicolumn{2}{|c|}{ Media } & \multicolumn{2}{|c|}{ Desv.Est } \\
\hline \multicolumn{2}{|c|}{ Adaptabilidad pre- ad } & \multicolumn{2}{|c|}{43.64} & 5.68 & \\
\hline CoefVar & Mínimo & Q1 & Mediana & Q3 & Máximo \\
\hline 13.01 & 39.00 & 40.00 & 41.00 & 47.00 & 56.00 \\
\hline Modo & $\mathrm{N}$ para $\mathrm{m}$ & & Asimetría & & tosis \\
\hline 40 & 4 & & 1.39 & & 66 \\
\hline
\end{tabular}

Fuente. Elaboración Propia. 
Tabla 21. Determinación de los datos con estadística descriptiva (adaptabilidad, post test administrativos)

\begin{tabular}{|c|c|c|c|c|c|}
\hline \multicolumn{6}{|c|}{ Estadísticos descriptivos: adaptabilidad post-ad } \\
\hline \multicolumn{3}{|l|}{ Variable } & Media & \multicolumn{2}{|l|}{ Desv.Est } \\
\hline \multicolumn{2}{|c|}{ Adaptabilidad pre- ad } & & 58.29 & 4.20 & \\
\hline CoefVar & Mínimo & Q1 & Mediana & Q3 & Máximo \\
\hline 7.20 & 55.00 & 55.00 & 57.00 & 60.00 & 69.00 \\
\hline Modo & $\mathrm{N}$ para $\mathrm{m}$ & & Asimetría & & osis \\
\hline 55 & 4 & & 1.64 & & 19 \\
\hline
\end{tabular}

Fuente. Elaboración Propia.

Con la dimensión adaptabilidad, al realizar la prueba de normalidad, se obtiene distribución no normal, por esta razón se utiliza la prueba de Wilcoxon Mann Whitney para comparar las medianas, se detalla en la tabla 22 líneas abajo.

Tabla 22. Prueba de Wilcoxon Mann Whitney

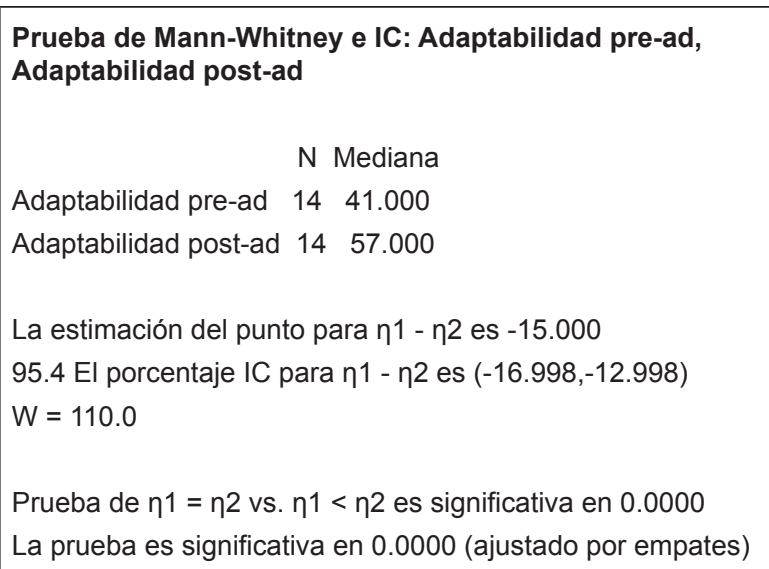

Fuente. Elaboración Propia.

Como se puede apreciar el nivel de significancia es igual a cero, entonces se rechaza la hipótesis nula y se acepta a hipótesis alternativa.

$H_{0}: \mu 1=\mu 2, H_{1}: \mu 1<\mu 2$, entonces la mediana de adaptabilidad pre test es menor que la mediana de adaptabilidad post test. Por lo tanto, hay evidencia suficiente para aceptar la hipótesis específica 3.

Como ambas distribuciones son normales se aplica la t- pareada (t-student) para comparar las mediciones antes y después de aplicar las estrategias. Esto se puede apreciar en la tabla 26 que se detalla líneas abajo.
Tabla 23. Área operacional antes y después de la estrategia (adaptabilidad, pre test y post test)

\begin{tabular}{|c|c|c|}
\hline Colaboradores & $\begin{array}{c}\text { Adaptabilidad } \\
\text { pre-op }\end{array}$ & $\begin{array}{c}\text { Adaptabilidad } \\
\text { post-op }\end{array}$ \\
\hline 1 & 41 & 57 \\
\hline 2 & 48 & 53 \\
\hline 3 & 56 & 69 \\
\hline 4 & 44 & 57 \\
\hline 5 & 47 & 62 \\
\hline 6 & 42 & 57 \\
\hline 7 & 41 & 56 \\
\hline 8 & 54 & 61 \\
\hline 9 & 54 & 64 \\
\hline 10 & 44 & 59 \\
\hline 11 & 47 & 62 \\
\hline 12 & 44 & 59 \\
\hline 13 & 49 & 64 \\
\hline 14 & 38 & 54 \\
\hline 15 & 41 & 55 \\
\hline 16 & 40 & 55 \\
\hline 17 & 45 & 60 \\
\hline 18 & 49 & 62 \\
\hline 19 & 41 & 56 \\
\hline 20 & 50 & 62 \\
\hline 21 & 47 & 56 \\
\hline 22 & 46 & 60 \\
\hline 23 & 49 & 62 \\
\hline 24 & 44 & 57 \\
\hline 25 & 54 & 68 \\
\hline 26 & 43 & 43 \\
\hline 27 & 53 & 66 \\
\hline 28 & 37 & 52 \\
\hline 29 & 48 & 57 \\
\hline
\end{tabular}

Fuente. Elaboración Propia.

Tabla 24. Determinación de los datos con estadística descriptiva (adaptabilidad, pre test operaciones)

\begin{tabular}{|lccccc|}
\hline \multicolumn{4}{ll}{ Estadísticos descriptivos: Adaptabilidad pre-op } \\
Variable & & Media & Desv.Est & \\
Adaptabilidad pre- op & 46.069 & 5.063 & \\
& & & & & \\
CoefVar & Mínimo & Q1 & Mediana & Q3 & Máximo \\
10.99 & 37.00 & 41.50 & 46.00 & 49.00 & 56.00 \\
& & & & & \\
Modo & N para moda & Asimetría & Curtosis \\
41,44 & 4 & 0.24 & & -0.67 \\
\hline
\end{tabular}

Fuente. Elaboración Propia. 
Tabla 25. Determinación de los datos con estadística descriptiva (adaptabilidad, post test operaciones)

\begin{tabular}{|lccccc|}
\hline \multicolumn{4}{ll}{ Estadísticos descriptivos: Adaptabilidad post-ad } \\
Variable & & Media & Desv.Est & \\
Adaptabilidad post - op & 56.414 & 5.220 & \\
& & & & & \\
CoefVar & Mínimo & Q1 & Mediana & Q3 & Máximo \\
9.25 & 43.00 & 52.00 & 56.00 & 61.50 & 63.00 \\
& & & & & \\
Modo & N para moda & Asimetría & Curtosis \\
55,63 & 4 & -0.57 & -0.14 \\
\hline
\end{tabular}

Fuente. Elaboración Propia.

Tabla 26. Prueba de $t$-student

Límite superior $95 \%$ para la diferencia de la media: -11.565

Prueba $\mathrm{t}$ de diferencia media $=0($ vs. $<0):$ Valor $\mathrm{T}=-17.98$ Valor $p=0.0$

Fuente. Elaboración Propia.

Como se puede apreciar el $p$ value es igual a cero, entonces por lo tanto es menor que 0.05 , entonces se rechaza la hipótesis nula y se acepta a hipótesis alternativa.

$\mathrm{H}_{\mathrm{o}}: \mu 1=\mu 2, \quad \mathrm{H}_{1}: \mu 1<\mu 2$, entonces la media de consistencia pre test es menor que la media del involucramiento post test. Por lo tanto, hay evidencia suficiente para aceptar la hipótesis específica 3 , porque se cumple tanto para los colaboradores administrativos y de operaciones.

4. Hipótesis específica 4: Mediante la implementación de los talleres de motivación en el trabajo se logrará la mejora de la dimensión misión de la cultura organizacional según el modelo de Denison en Servicios Analíticos Generales S.A.C.

En las Tablas 27 y 31 se puede encontrar los valores de las encuestas realizadas al área de administración y de operaciones respectivamente de la dimensión misión. Se aplica en el Minitab los datos del pre test y post test para los estadísticos descriptivos para el área administrativa (Tabla 28 y Tabla 29) y de operaciones (Tabla 32 y Tabla 33).

Como ambas distribuciones son normales se aplica la t- pareada (t-student) para comparar las mediciones antes y después de aplicar las estrategias. Esto se puede apreciar en la tabla 30 que se detalla líneas abajo.
Tabla 27. Área administrativa antes y después de la estrategia (misión, pre test y post test)

\begin{tabular}{|c|c|c|}
\hline Colaboradores & Misión pre-ad & Misión post-ad \\
\hline 1 & 54 & 65 \\
\hline 2 & 36 & 53 \\
\hline 3 & 60 & 71 \\
\hline 4 & 44 & 59 \\
\hline 5 & 52 & 67 \\
\hline 6 & 39 & 54 \\
\hline 7 & 45 & 60 \\
\hline 8 & 50 & 63 \\
\hline 9 & 44 & 59 \\
\hline 10 & 50 & 63 \\
\hline 11 & 44 & 59 \\
\hline 12 & 76 & 61 \\
\hline 13 & 43 & 58 \\
\hline 14 & 54 & 61 \\
\hline
\end{tabular}

Fuente. Elaboración Propia.

Tabla 28. Determinación de los datos con estadística descriptiva (misión, pre test administrativos)

\begin{tabular}{|c|c|c|c|c|c|}
\hline \multicolumn{6}{|c|}{ Estadísticos descriptivos: Misión pre-ad } \\
\hline \multicolumn{3}{|l|}{ Variable } & Media & \multicolumn{2}{|c|}{ Desv.Est } \\
\hline \multicolumn{2}{|c|}{ Misión pre- ad } & & 49.36 & 10.02 & \\
\hline CoefVar & Mínimo & Q1 & Mediana & Q3 & Máximo \\
\hline 20.30 & 36.00 & 43.75 & 47.50 & 54.00 & 76.00 \\
\hline Modo & $\mathrm{N}$ para $\mathrm{r}$ & & Asimetría & $\mathrm{Cu}$ & tosis \\
\hline 44 & 3 & & 1.43 & & 02 \\
\hline
\end{tabular}

Fuente. Elaboración Propia.

Tabla 29. Determinación de los datos con estadística descriptiva (misión, post test administrativos)

\begin{tabular}{|lccccc|}
\hline \multicolumn{4}{l}{$\begin{array}{l}\text { Estadísticos descriptivos: } \\
\text { Variable }\end{array}$} & \multicolumn{4}{l}{ Misión post-ad } \\
Misión post - ad & & 60.93 & 4.76 & \\
& & & & & \\
CoefVar & Mínimo & Q1 & Mediana & Q3 & Máximo \\
7.82 & 53.00 & 58.75 & 60.50 & 63.50 & 71.00 \\
& & & & & \\
Modo & N para moda & Asimetría & Curtosis \\
59 & 3 & 0.38 & 0.52 \\
\hline
\end{tabular}

Fuente. Elaboración Propia.

Tabla 30. Prueba de t-student

Límite superior $95 \%$ para la diferencia de la media: -7.76

Prueba t de diferencia media $=0$ (vs. $<0):$ Valor $\mathrm{T}=-5.38$

Valor $p=0.0$

Fuente. Elaboración Propia. 
Como se puede apreciar el valor de $p$ es igual a cero, entonces se rechaza la hipótesis nula y se acepta a hipótesis alternativa. Ho: $\mu 1=\mu 2, \mathrm{H} 1: \mu 1$ $<\mu 2$, entonces la media de misión pre test es menor que la media de misión post test. Por lo tanto, hay evidencia suficiente para aceptar la hipótesis específica 4 para los administrativos.

Tabla 31. Área operacional antes y después de la estrategia (misión, pre test y post test)

\begin{tabular}{|c|c|c|}
\hline Colaboradores & Misión pre-op & Misión post-op \\
\hline 1 & 46 & 57 \\
\hline 2 & 43 & 58 \\
\hline 3 & 60 & 68 \\
\hline 4 & 50 & 66 \\
\hline 5 & 43 & 57 \\
\hline 6 & 44 & 59 \\
\hline 7 & 40 & 55 \\
\hline 8 & 65 & 68 \\
\hline 9 & 51 & 63 \\
\hline 10 & 45 & 60 \\
\hline 11 & 49 & 62 \\
\hline 12 & 44 & 59 \\
\hline 13 & 67 & 68 \\
\hline 14 & 39 & 54 \\
\hline 15 & 50 & 59 \\
\hline 16 & 47 & 59 \\
\hline 17 & 44 & 59 \\
\hline 18 & 47 & 58 \\
\hline 19 & 45 & 59 \\
\hline 20 & 37 & 52 \\
\hline 21 & 44 & 57 \\
\hline 22 & 53 & 63 \\
\hline 23 & 67 & 69 \\
\hline 24 & 44 & 59 \\
\hline 25 & 65 & 67 \\
\hline 26 & 54 & 54 \\
\hline 27 & 47 & 62 \\
\hline 28 & 35 & 52 \\
\hline 29 & 37 & 53 \\
\hline
\end{tabular}

Fuente. Elaboración Propia.

Tabla 32. Determinación de los datos con estadística descriptiva (misión, pre test operaciones)

\begin{tabular}{|lccccc|}
\hline \multicolumn{4}{ll}{ Estadísticos descriptivos: Misión pre-op } \\
Variable & & & \\
Misión pre- op & & 48.34 & 4.965 & \\
& & & & & \\
CoefVar & Mínimo & Q1 & Mediana & Q3 & Máximo \\
8.30 & 52.00 & 57.00 & 59.00 & 63.00 & 69.00 \\
& & & & & \\
Modo & N para moda & Asimetría & Curtosis \\
59 & 7 & 0.39 & -0.62 \\
\hline
\end{tabular}

Fuente. Elaboración Propia.
Tabla 33. Determinación de los datos con estadística descriptiva (misión, post test operaciones)

\begin{tabular}{|c|c|c|c|c|c|}
\hline \multicolumn{6}{|c|}{ Estadísticos descriptivos: Misión post-op } \\
\hline \multicolumn{3}{|l|}{ Variable } & Media & \multicolumn{2}{|l|}{ Desv.Est } \\
\hline \multicolumn{2}{|c|}{ Misión post - op } & \multicolumn{2}{|c|}{59.828} & \multicolumn{2}{|l|}{4.817} \\
\hline CoefVar & Mínimo & Q1 & Mediana & Q3 & Máximo \\
\hline 8.07 & 52.00 & 57.00 & 59.00 & 63.00 & 69.00 \\
\hline Modo & \multicolumn{2}{|c|}{$\mathrm{N}$ para moda } & Asimetría & \multicolumn{2}{|c|}{ Curtosis } \\
\hline 59 & \multicolumn{2}{|l|}{7} & -0.33 & \multicolumn{2}{|c|}{-0.58} \\
\hline
\end{tabular}

Fuente. Elaboración Propia.

Con la dimensión misión, al realizar la prueba de normalidad, se obtiene distribución no normal, por esta razón se utiliza la prueba de Wilcoxon Mann Whitney para comparar las medianas, se detalla en el tabla 34 líneas abajo.

Tabla 34. Prueba de Wilcoxon Mann Whitney

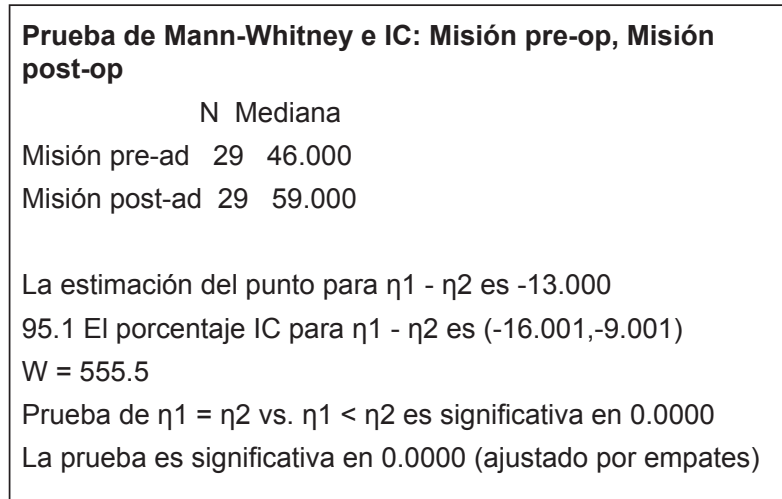

Fuente. Elaboración Propia.

Como se puede apreciar el nivel de significancia es igual a cero, entonces se rechaza la hipótesis nula y se acepta a hipótesis alternativa.

$H_{0}: \mu 1=\mu 2, H_{1}: \mu 1<\mu 2$, entonces la mediana de misión pre test es menor que la mediana de misión post test. Por lo tanto, hay evidencia suficiente para aceptar la hipótesis específica 4 porque se cumple tanto para colaboradores administrativos y de operaciones.

5. Hipótesis General: Mediante la implementación de las estrategias de liderazgo y motivación se logrará la mejora de la cultura organizacional en Servicios Analíticos Generales S.A.C.

Hay evidencia suficiente para aceptar las 4 hipótesis específicas, por lo tanto la hipótesis general también se acepta. 


\section{CONCLUSIONES}

a. El taller de motivación fue importante iniciar conociéndose entre los colaboradores, porque a pesar de algunos que tenían años trabajando no habían cruzado palabra alguna, lo que se puede concluir que había un aislamiento entre el personal administrativo y de operaciones.

b. En un trabajo en equipo la comunicación es más fluida entre todos los colaboradores del grupo de trabajo.

c. La dimensión adaptabilidad presenta una distribución no normal, en referencia al personal administrativo, lo que indica que para un grupo humano pequeño adaptarse a un cambio es difícil.

d. El personal de operaciones es muy sensible al cambio, por lo general son tímidos y no expresan de manera correcta sus ideas a diferencia de los administrativos.

e. La percepción del personal está cambiando y esto ayudará al cambio en momentos de turbulencia.

\section{RECOMENDACIONES}

a. Es muy importante activar la motivación intrínseca en el personal, porque la persona está apasionada por lo que hace.

b. Crear equipos de líderes con tareas sencillas en un inicio para el personal que no sea proactivo, para elevar su confianza y responsabilidad. Los líderes que se formen deben ser coherentes con sus palabras y hechos.

c. Al realizar capacitaciones de cultura organizacional con mayor frecuencia, se ampliaría para todo el personal así sería más sencillo que el personal se sienta identificado con la organización.

\section{REFERENCIAS BIBLIOGRÁFICAS}

Barreto, L. y Bonilla, M. (2011). Perfil cultural y desempeño organizacional en la empresa Ecocapital internacional S.A. (Tesis de Maestría, Universidad de la Sabana). Recuperado de http://intellectum.unisabana.edu.co/bitstream/ handle/10818/171/Ligia \%20Marcela \%20 Barreto\%20Pardo.pdf?sequence=1.
Bonavia, T., Prado, V. y García-Hernández A. (2010). Adaptación al español del instrumento sobre cultura organizacional de Denison. SUMMA Psicológica UST. 7 (1), 15 - 32. Recuperado de https://dialnet.unirioja.es/ servlet/articulo?codigo $=3294911$

Bower, K. (2000). Scientific Computing \& Instrumentation. Recuperado de https://www. minitab.com/uploadedFiles/Content/News/ Published_Articles/paired_t_test.pdf

Calderón, G., Murillo, S. y Torres, K. (2003). Cultura organizacional y bienestar laboral. Cuad. Adm. 16 (25), 109-137. Recuperado de http://www. javeriana.edu.co/Facultades/C_Econom_y_ Admon/cuadernos_admon/pdfs/5_25.pdf

Ciencia y educación, (2016). ¿ “P=0.05" sinónimo de descubrimiento científico? Recuperado de https://www.mimetikoss.com/blogs/blogbiomimetico/114951364-p-0-05-sinonimo-dedescubrimiento-cientifico

De la Garza, J., Morales. B. y González B., (2013). Análisis Estadístico Multivariante. México: McGraw-Hill.

Denison, D. (1991). Cultura Corporativa. Santafé de Bogotá: Legis

Denison, D. R. (2001). Organizational culture: can it be a key lever for driving organizational change? En C. L. Cooper, S. Cartwright y P. Ch. Earley (Eds.), The International Handbook of Organizational Culture and Climate (pp. 347372). Chichester: John Wiley \& Sons.

Hernández, R., Fernández, C. y Baptista, P. (2010). Metodología de la investigación. 5ta edición. México: Editorial McGraw-Hill/Interamericana Editores S.A. de C.V.

Hospinal, S. (2014). Clima organizacional y satisfacción laboral en el área de producción de FYD Inversiones S.A.C. (Tesis inédita de maestría). UNMSM, Lima, Perú.

Montgomery, D. (2015). Diseño y análisis de experimentos. 2da Edición. México: Editorial Limusa, S.A.

Quintero N., Africano, N. y Faría, E. (2008). Clima Organizacional y Desempeño Laboral del Personal. Revista NEGOTIUM. 3 (9), 33-51. Recuperado de http://www.revistanegotium. org.ve/pdf/9/Art2.pdf.

Sánchez, H. y Reyes, C. (2006). Metodología y diseño en la investigación científica. 4ta Edición. Lima: Editorial Visión Universitaria. 
Servicios Analíticos Generales S.A.C. (2016). Porque nos preocupa la vida, atendemos el Medio Ambiente. Recuperado de http://www. sagperu.com/
Siegel, S. \& Castellan, N. J. (1995). Estadística no paramétrica, aplicada a las ciencias de la conducta. México: Editorial Trillas. 
